\title{
Magnetic structure and spin dynamics of the quasi-one-dimensional spin-chain antiferromagnet $\mathrm{BaCo}_{2} \mathrm{~V}_{2} \mathrm{O}_{8}$
}

\author{
Yu Kawasaki, ${ }^{1,2}$ Jorge L. Gavilano, ${ }^{1}$ Lukas Keller, ${ }^{1}$ Jürg Schefer, ${ }^{1}$ Niels Bech Christensen,,${ }^{1,3}$ Alex Amato, ${ }^{4}$ Takashi Ohno, ${ }^{2}$ \\ Yutaka Kishimoto, ${ }^{2}$ Zhangzhen He, ${ }^{5,6}$ Yutaka Ueda, ${ }^{5}$ and Mitsuru Itoh ${ }^{7}$ \\ ${ }^{1}$ Laboratory for Neutron Scattering PSI, CH-5232 Villigen PSI, Switzerland \\ ${ }^{2}$ Institute of Technology and Science, The University of Tokushima, Tokushima 770-8506, Japan \\ ${ }^{3}$ Materials Research Division, Ris $\phi$ National Laboratory for Sustainable Energy, Technical University of Denmark, \\ Frederiksborgvej 399 P.O. Box 49 DK-4000 Roskilde \\ ${ }^{4}$ Laboratory for muon Spin Spectroscopy, ETH Zürich and PSI, CH-5232 Villigen PSI, Switzerland \\ ${ }^{5}$ Institute for Solid State Physics, Univ. of Tokyo, Chiba, Japan \\ ${ }^{6}$ Fujian Institute of Research on the Structure of Matter, Chinese Academy of Sciences, Fuzhou, Fujian 350002, P. R. China \\ ${ }^{7}$ Materials and Structures Laboratory, Tokyo Institute of Technology, Yokohama, Japan
}

(Received 27 October 2010; revised manuscript received 18 December 2010; published 25 February 2011)

\begin{abstract}
We report a neutron diffraction and muon spin relaxation $\mu$ SR study of static and dynamical magnetic properties of $\mathrm{BaCo}_{2} \mathrm{~V}_{2} \mathrm{O}_{8}$, a quasi-one-dimensional spin-chain system. A proposed model for the antiferromagnetic structure includes: a propagation vector $\vec{k}_{\mathrm{AF}}=(0,0,1)$, independent of external magnetic fields for fields below a critical value $H_{c}(T)$. The ordered moments of $2.18 \mu_{B}$ per Co ion are aligned along the crystallographic $c$ axis. Within the screw chains, along the $c$ axis, the moments are arranged antiferromagnetically. In the basal planes the spins are arranged ferromagnetically (forming zigzag paths) along one of the axes and antiferromagnetically along the other. The temperature dependence of the sublattice magnetization is consistent with the expectations of the three-dimensional (3D) Ising model. A similar behavior is observed for the internal static fields at different muon stopping sites. Muon time spectra measured at weak longitudinal fields and temperatures much higher than $T_{N}$ can be well described using a single muon site with an exponential muon spin relaxation that gradually changes into an stretched exponential on approaching $T_{N}$. The temperature-induced changes of the relaxation suggest that the Co fluctuations dramatically slow down and the system becomes less homogeneous as it approaches the antiferromagnetic state.
\end{abstract}

DOI: 10.1103/PhysRevB.83.064421

PACS number(s): 75.10.Pq, 75.25.-j, 75.40.Cx, 75.40.Gb

\section{INTRODUCTION}

One-dimensional (1D) spin-chain systems with nearestneighbor antiferromagnetic (AF) exchange interactions have attracted much attention in the last decades because they display a rich variety of ground states with nonclassical magnetic phenomena. ${ }^{1-3}$ It is well known that an ideal 1D AF spin system does not undergo long-range order (LRO) at finite temperatures due to strong quantum spin fluctuations. ${ }^{4}$ But slight perturbations of the system by, for instance, weak interchain interactions or doping with nonmagnetic impurities, can make the quantum critical state unstable and favors three-dimensional (3D) AF LRO.,

In $2005 \mathrm{BaCo}_{2} \mathrm{~V}_{2} \mathrm{O}_{8}$, a new member of the quasi-1D AF spin-chain family, was synthesized by Zhangzhen $\mathrm{He}$ and coworkers. ${ }^{7-9}$ They succeeded in growing large single crystals of high quality. $\mathrm{BaCo}_{2} \mathrm{~V}_{2} \mathrm{O}_{8}$ is an insulator with a very anisotropic magnetic susceptibility $\chi$; the easy axis of magnetization is along the crystallographic $c$ axis. The temperature dependence of the magnetic susceptibility $\chi(T)$ displays a broad maximum near $30 \mathrm{~K}$ signaling the development of $1 \mathrm{D}$ short-range order. ${ }^{7}$

Crystal-field effects and spin-orbit coupling partially lift the degeneracy of the single ion $\mathrm{Co}^{2+}\left(3 d^{7}\right)$ multiplet resulting in a doublet ground state. Thus the spin system may be considered as a collection of effective $S=1 / 2$ spin chains. ${ }^{10}$ With lowering temperature, a rapid drop of $\chi$ at $T_{N}=$ $5.4 \mathrm{~K}$ signals the onset of a long-range-ordered AF state at zero field. ${ }^{7}$ Quantum fluctuations of the spins strongly affect the ordered state, as evidenced by the phenomena observed in external fields applied parallel to the chains, which results in an unusual field-temperature phase diagram (see Fig. 1).

The magnetization curves $M(H)$ are strongly nonlinear. ${ }^{10-12}$ External magnetic fields $H$ above a critical value $H_{c}(T)$ suppress the $3 \mathrm{D}$ AF Néel state. At $T=0 \mathrm{~K}$, $\mu_{0} H_{c}=3.9 \mathrm{~T}$. The field-induced suppression of the Néel state occurs in an unusual manner. Above $1.8 \mathrm{~K}, H$ induces a reentrant phenomenon from the long-range 3D Néel state into a 1D quantum liquid state (order-disorder transition). ${ }^{13}$ This transition was theoretically predicted first for gapped spin systems doped with nonmagnetic impurities. ${ }^{14}$

At lower temperatures, however, external fields $H \geqslant H_{c}(T)$ result in the development of an incommensurably modulated AF state. The existence of this novel phase was demonstrated experimentally by Kimura and coworkers. ${ }^{15-18}$ They found that, for the modulated structure, a propagation vector $\vec{k}_{M}=$ $(0,0,1+\delta)$ with $\delta$ tunable by the external magnetic field.

Although many interesting properties have been investigated in $\mathrm{BaCo}_{2} \mathrm{~V}_{2} \mathrm{O}_{8}$, there are no reports on the details of the structure of the Néel ordered state; [i.e., in external magnetic fields $H<H_{c}(T)$ ]. In addition, there is very little information about the spin dynamics in this system, except for two NMR studies. ${ }^{19,20}$ We present here a detailed model for the magnetic structure, obtained from neutron diffraction measurements in powder samples and in single crystals of this material. We also report the results of $\mu \mathrm{SR}$ measurements below and above $T_{N}$. The latter shine light on the dynamics of the spin system. 


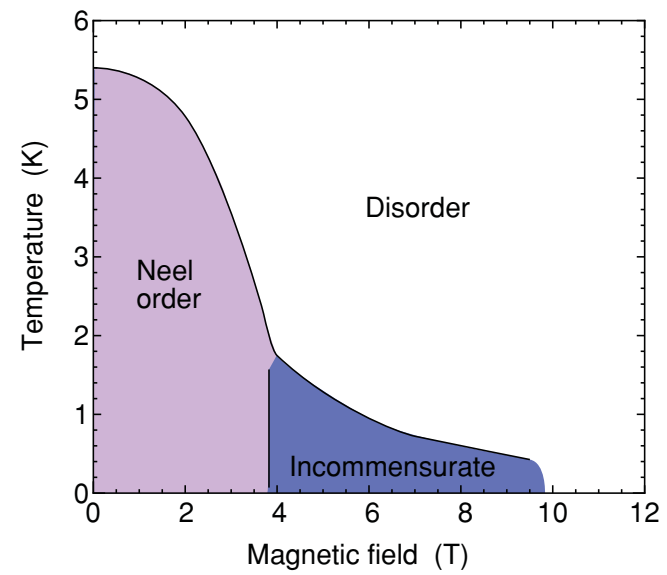

FIG. 1. (Color online) Schematic representation of the magneticfield-Temperature phase diagram of $\mathrm{BaCo}_{2} \mathrm{~V}_{2} \mathrm{O}_{8}$ for fields applied along the $c$ axis. Two magnetically ordered phases are distinguished: (i) The Néel state; a 3D AF state at low fields, and (ii) an incommensurably modulated phase at higher fields (from Kimura and coworkers ${ }^{17}$ ).

The physical properties of $\mathrm{BaCo}_{2} \mathrm{~V}_{2} \mathrm{O}_{8}$ can be understood in the context of Ising spin chains with weak interchain interactions as described by the $S=1 / 2 \mathrm{XXZ}$ model where a single chain in the presence of an external magnetic field $H$ is described by the Hamiltonian

$\mathcal{H}=J \sum_{i}\left[S_{i}^{z} S_{i+1}^{z}+\epsilon\left(S_{i}^{x} S_{i+1}^{x}+S_{i}^{y} S_{i+1}^{y}\right)\right]-g \mu_{B} \sum_{i} S_{i}^{z} H$.

From magnetization and electron spin resonance (ESR) data it has been determined for $\mathrm{BaCo}_{2} \mathrm{~V}_{2} \mathrm{O}_{8}$ that the $\mathrm{AF}$ exchange interaction is $J / k_{B}=65 \mathrm{~K}$, the anisotropy parameter $\epsilon \approx 0.5$, and $g \approx 6.2$. The interchain interaction $J^{\prime}$ is of the order of $J^{\prime} / J \approx 0.06 .^{11}$ In this model, Néel order occurs at zero field with a nonvanishing gap. ${ }^{5}$ The application of external magnetic fields, $H>H_{c}$, restores the Tomonaga-Luttinger spin-liquid state with longitudinal and transversal incommensurate spin fluctuations that characterize 1D Ising systems. ${ }^{2,21}$

$\mathrm{BaCo}_{2} \mathrm{~V}_{2} \mathrm{O}_{8}$ crystallizes adopting a tetragonal structure with space-group symmetry $I 41 / a c d$, room-temperature lattice parameters of $a=12.444 \AA$ and $c=8.415 \AA$, and 8 formula units per unit cell. All the magnetic Co ions are equivalent. They lie in the centers of $\mathrm{CoO}_{6}$ octahedra, and have strong $\mathrm{AF}$ exchange interactions with $\mathrm{Co}$ ions in the neighboring edge-sharing $\mathrm{CoO}_{6}$ octahedra. The $\mathrm{Co}^{2+}$ spins, here referred simply as "spins," form a collection of screwtype chain structures along the $c$ axis. The screw chains are separated by nonmagnetic $\mathrm{VO}_{4}\left(\mathrm{~V}^{5+}\right)$ and $\mathrm{BaO}_{4}\left(\mathrm{Ba}^{2+}\right)$ tetrahedra, resulting in a quasi-one-dimensional structural arrangement of the spins with a strong Ising-type magnetic anisotropy. A representation of the crystal structure is given in Fig. 2.

\section{EXPERIMENTAL PROCEDURE}

Polycrystalline samples of $\mathrm{BaCo}_{2} \mathrm{~V}_{2} \mathrm{O}_{8}$ were synthesized by a standard solid-state reaction method using high-purity

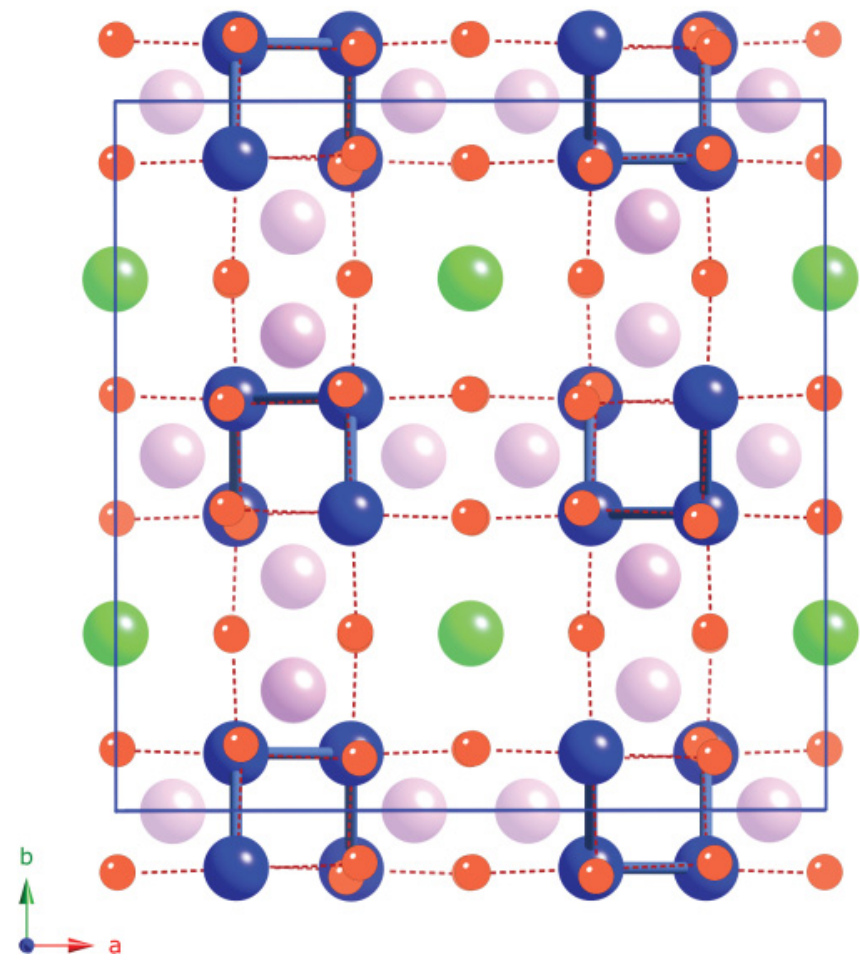

FIG. 2. (Color online) Representation of the basal plane projection of the crystal structure of $\mathrm{BaCo}_{2} \mathrm{~V}_{2} \mathrm{O}_{8}$. This figure emphasizes large blue circles, representing Co ions with the rods joining them representing the nearest-neighbor $\mathrm{Co}-\mathrm{Co}$ bonds. The small orange circles represent the $\mathrm{O}$ ions, the green and violet circles represent the $\mathrm{Ba}$ and $\mathrm{V}$ ions, respectively.

reagents of $\mathrm{BaCO}_{3}(4 \mathrm{~N}), \mathrm{CoC}_{2} \mathrm{O}_{4} \cdot 2 \mathrm{H}_{2} \mathrm{O}(3 \mathrm{~N})$ and $\mathrm{V}_{2} \mathrm{O}_{5}$ (4N) as starting materials. Single crystals of $\mathrm{BaCo}_{2} \mathrm{~V}_{2} \mathrm{O}_{8}$ (of dimensions of the order of $10 \times 8 \times 3 \mathrm{~mm}^{3}$ ) were grown by a spontaneous nucleation method from the same starting materials. Details of the material synthesis have been previously described. ${ }^{7,8,12}$ Our material was characterized by x-ray diffraction and magnetic susceptibility. The crystal structure and the magnetic properties confirmed the conclusions based on previous data. ${ }^{13}$

The magnetic structure of $\mathrm{BaCo}_{2} \mathrm{~V}_{2} \mathrm{O}_{8}$ in zero external magnetic field was investigated by measuring neutron powder diffraction patterns at temperatures down to $1.8 \mathrm{~K}$ using incident neutrons of wavelength of $2.4503 \AA$ in the cold neutron powder diffractometer DMC at SINQ, Paul Scherrer Institute (PSI), Switzerland. The sample consisted of $8 \mathrm{~g}$ of powdered $\mathrm{BaCo}_{2} \mathrm{~V}_{2} \mathrm{O}_{8}$ material enclosed in a thin-walled $\mathrm{V}$ cylindrical container. On a single-crystalline sample, we measured the temperature and field dependencies of some selected magnetic Bragg reflections using the triple axis spectrometer Rita-II and the thermal single crystal diffractometer TriCS, both at SINQ, PSI (the latter with incident neutrons of wavelength $\lambda=1.18 \AA$ ). External magnetic fields of up to $\mu_{0} H=4.5 \mathrm{~T}$ were applied along $c$ axis. $\mu$ SR time spectra were measured on a polycrystalline sample, in the temperature range between 1.5 and $100 \mathrm{~K}$ in zero and weak longitudinal magnetic fields, using the general purpose surface-muon instrument GPS at the Swiss Muon Source of PSI. 


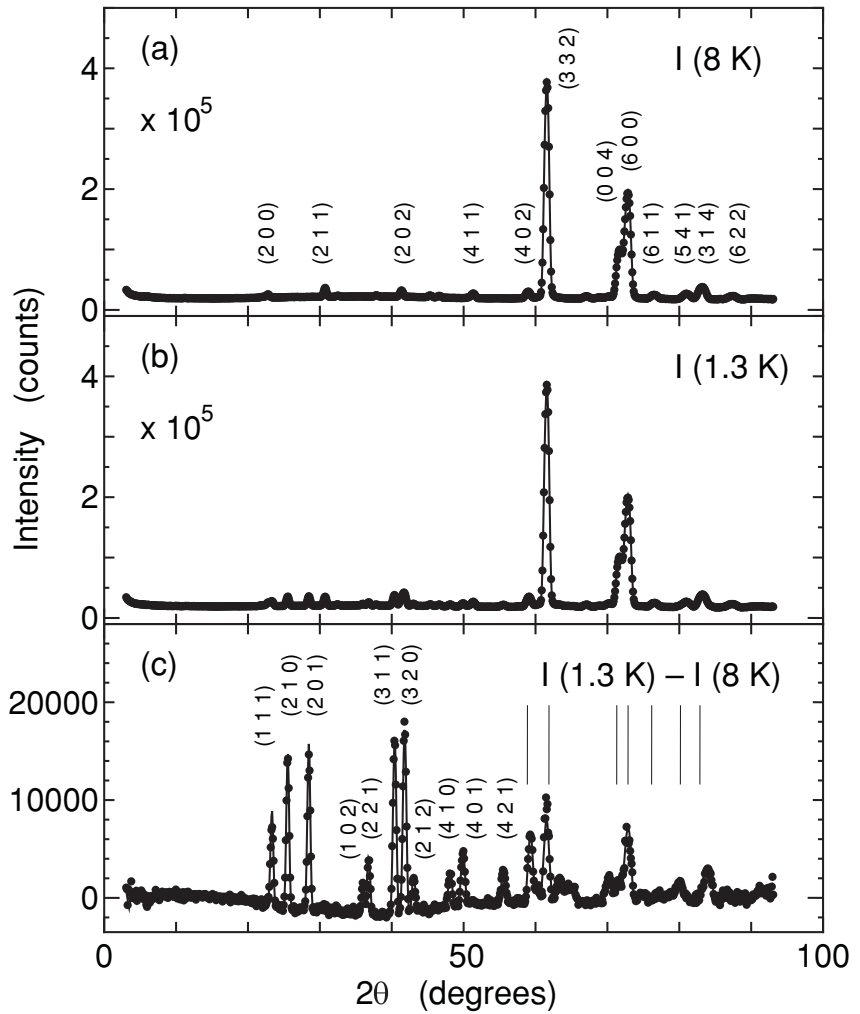

FIG. 3. Bragg scattering profiles for $\mathrm{BaCo}_{2} \mathrm{~V}_{2} \mathrm{O}_{8}$ at $T=8 \mathrm{~K}$ (a) and $T=1.3 \mathrm{~K}(\mathrm{~b})$. A pattern corresponding to the difference of intensities $[I(1.3 \mathrm{~K})-I(8 \mathrm{~K})]$ and revealing only the magnetic Bragg reflections is shown in (c). The solid lines represent the refinement of the data using a model described in the text.

\section{RESULTS AND DISCUSSION}

\section{A. Magnetic structure}

In Figs. 3(a) and 3(b) we display neutron powder diffraction patterns above $(8 \mathrm{~K})$ and below $(1.3 \mathrm{~K})$ the Néel temperature, respectively. The magnetic Bragg reflections [i.e., the difference of intensities $I(1.3 \mathrm{~K})-I(8 \mathrm{~K})]$ is displayed in Fig. 3(c). A refinement using the FULLPROF software package ${ }^{22}$ yielded a very good fit of all the magnetic and nonmagnetic reflections. From the nuclear reflections we obtained for the lattice parameters of the tetragonal cell at $8 \mathrm{~K}: a=12.389 \AA$ and $c=8.375 \AA$. All the magnetic reflections, which appear below $T_{N}$, could be indexed in the tetragonal system with a wave vector $\vec{k}_{\mathrm{AF}}=(0,0,1)$. The best fits to the data are represented by solid lines in Figs. 3(a)-3(c), where the conventional Rietveld factors are $R_{\mathrm{p}}=6.08, R_{\mathrm{wp}}=6.13$ and $R_{\exp }=1.13$.

The refined spin structure is represented in Fig. 4, where the Co ions are depicted as circles and their bonds as the heavy solid lines joining them. Different coordinates along the $c$ axis are indicated by the different fillings of the circles. This figure emphasizes the parts of the screw chains contained within the unit cell. The chains run along the $c$ axis, the ordered Co moments are aligned along the same direction and " $u$ " and " $d$ " represent up and down spin directions, respectively. The spins are arranged antiferromagnetically along the screw chains, but at a given basal plane they are arranged antiferromagnetically

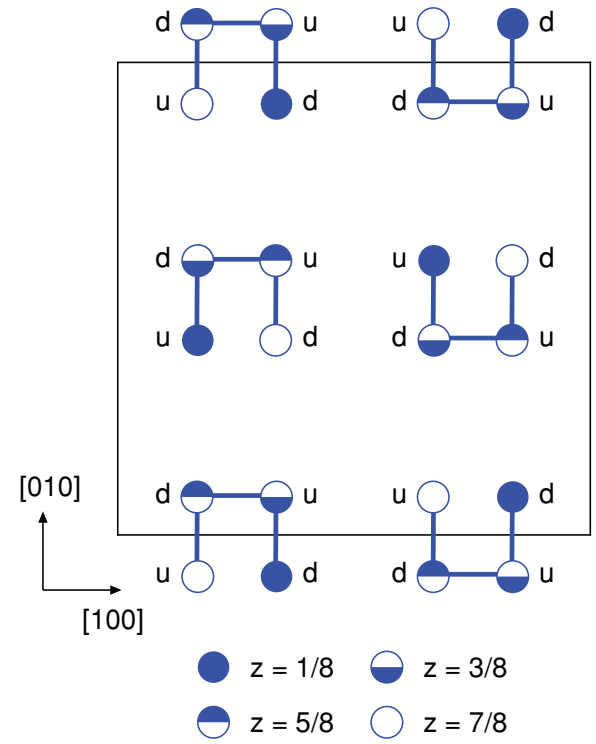

FIG. 4. (Color online) Representation of the magnetic structure of $\mathrm{BaCo}_{2} \mathrm{~V}_{2} \mathrm{O}_{8}$ in the AF state. Co ions are depicted as circles and their bonds as solid lines. The quasi-one-dimensional screw-type spin chains run along the $c$ axis, and the "up" and "down" spin directions are represented by "u" and "d," respectively.

(ferromagnetically) forming zigzag paths along the $a(b)$ axis. The Bragg peaks are very narrow, resolution limited; thus, the 3D long-range character of the AF order is confirmed. From the refinement, the magnitude of Co moments is estimated to be $2.18 \mu_{\mathrm{B}}$, which for an effective spin $S=1 / 2$ implies an effective $g$ factor of $g \approx 4.4$.

Our neutron powder-diffraction data are not compatible with the value $g=6.2$, obtained from high-field ESR and magnetization results. ${ }^{7}$ Assuming the ESR $g$ value, the calculated intensities of the magnetic reflections would be too large by a factor of 2 to fit the experimental data. Therefore, we conclude that the Néel state involves reduced Co moments. This reduction is probably due to quantum fluctuations expected in the 3D XXZ model. But the discrepancy of the $g$ factors extracted from neutron scattering, high-field ESR and magnetization results have to be considered carefully since these techniques probe the spin system under different conditions.

We have measured the temperature and field dependencies of selected magnetic Bragg reflections on a single-crystalline sample using Rita-II. A summary of these results is presented in Fig. 5. The circles in the main panel represent the square root of the integrated magnetic Bragg intensity $\sqrt{I_{B}}$ of the $(3,2,0)$ reflection as a function of temperature. This quantity is proportional to the sublattice magnetization. Its functional form [i.e., the $\sqrt{I_{B}}(T)$ curve] follows the expectations for a 3D Ising antiferromagnet, here represented by the solid line. In a limited temperature range near $T_{N}\left(0.8 \leqslant T / T_{N} \leqslant 1\right)$, we find

$$
\sqrt{I_{B}} \propto\left(1-T / T_{N}\right)^{\beta} .
$$

The fits to the data using Eq. (2) yielded a Néel temperature $T_{N}=5.4 \mathrm{~K}$, as expected, and a critical exponent $\beta=0.28$. This value is close to the predictions of the $3 \mathrm{D}$ Ising model, 


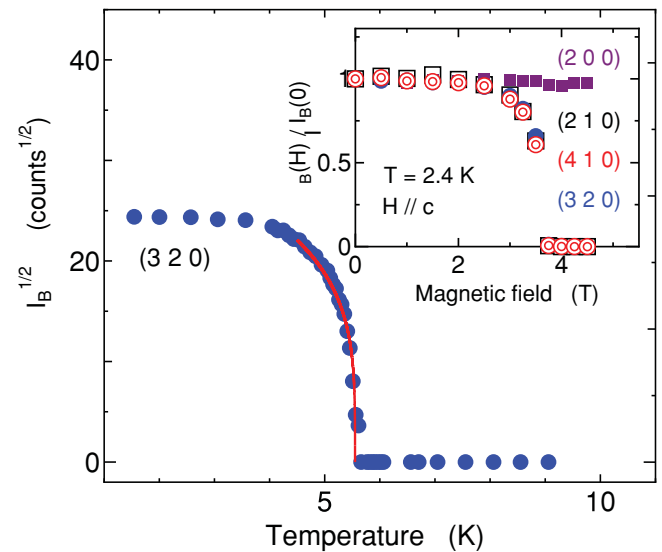

FIG. 5. (Color online) The temperature dependence of the square root of the integrated intensity of the $(3,2,0)$ magnetic reflection. The inset shows the magnetic field dependence of the Bragg intensity of the $(2,1,0),(4,1,0)$, and $(3,2,0)$ magnetic reflections and the nuclear $(2,0,0)$ as a function of magnetic field along the $c$ axis.

which has been estimated to be $\beta \approx 5 / 16$, with the exact value depending slightly on the approximations used. For the twodimensional (2D) case: $\beta=1 / 8 .^{23-26}$

The inset of Fig. 5 shows the normalized integrated intensity of the three magnetic Bragg reflections $(2,1,0),(4,1,0)$, and $(3,2,0)$ as a function of magnetic field along the $c$ axis. For comparison, we also plot the intensity of the nuclear Bragg reflection $(2,0,0)$. The data indicate that the magnetic ordering is suppressed by external magnetic fields of $3.75 \mathrm{~T}$ along the $c$ axis at $2.4 \mathrm{~K}$. This is in agreement with the results obtained from measurements of the specific heat in external magnetic fields. ${ }^{8}{ }^{11}$ We found no measurable field dependence for the magnetic propagation vector $k_{\mathrm{AF}}$ in the Néel ordered phase.

In an effort to shed more light on the nature of the phase transition into the spin liquid phase, we have searched for quasielastic scattering in the vicinity of the above-mentioned magnetic Bragg reflections. The searches were performed at temperatures near $T_{N}$ and zero external magnetic field using the thermal single crystal diffractometer TriCS with a $2 \mathrm{D}$ detector at SINQ, PSI. No clear quasielastic signals were detected.

\section{B. Muon spin relaxation and rotation}

To investigate the spin dynamics of $\mathrm{BaCo}_{2} \mathrm{~V}_{2} \mathrm{O}_{8}$ in the paramagnetic state, we have performed $\mu \mathrm{SR}$ experiments in zero-field $(\mathrm{ZF})$ and in weak longitudinal external magnetic fields (LF). ${ }^{27}$ Four typical $\mu$ SR spectra above $T_{N}$, measured in a field of 20 Oe, are shown in Fig. 6. This weak external field effectively suppresses the contribution to the muon relaxation due to quasistatic nuclear dipolar fields. This leaves basically a dynamical relaxation, which for the case of a single relaxation rate is usually well described by a simple exponential function. ${ }^{28}$ More complex dynamic cases are described using stretched exponential functions. ${ }^{29,30}$

The LF- $\mu$ SR time spectra data above $T_{N}$ were fit using

$$
G_{z}(t)=A \exp \left[-\left(\lambda_{\mathrm{LF}} t\right)^{n}\right],
$$

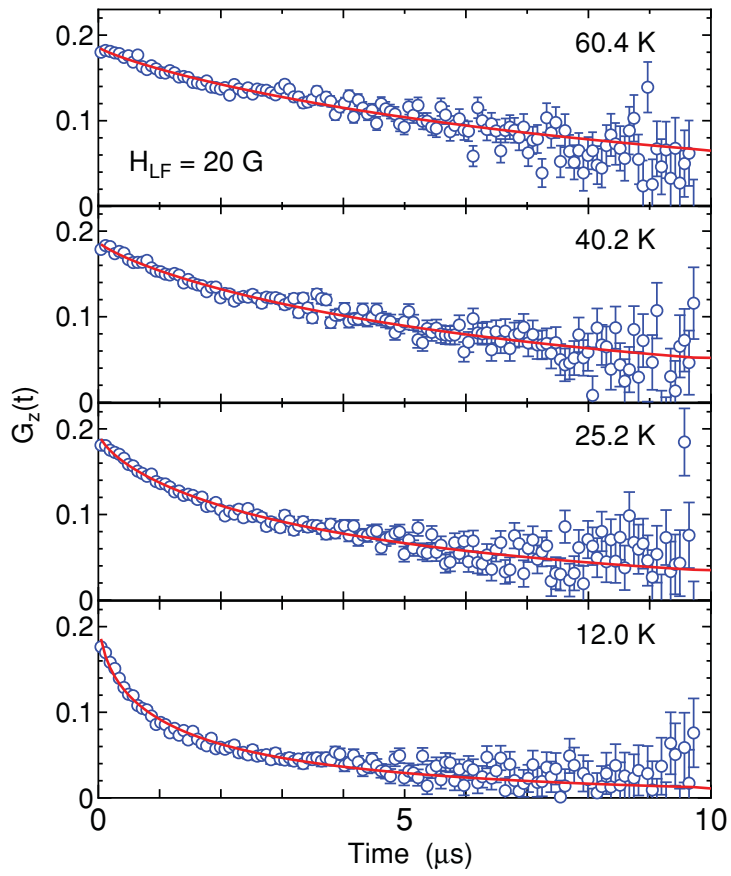

FIG. 6. (Color online) $\mu \mathrm{SR}$ time spectra measured in a weak longitudinal external magnetic field, of $20 \mathrm{G}$ in the paramagnetic state at four temperatures well above $T_{N}$. The solid lines represent fits to the data using effectively a single component (see text).

where $A$ is the initial asymmetry and $\lambda_{\mathrm{LF}}$ is a parameter also denoted here as the muon longitudinal spin relaxation rate. The best fits are represented by the solid lines in Fig. 6. In the paramagnetic state the relaxation (of dynamic character) is associated with the fluctuations of the Co moments. A stretched exponent $n \approx 1$ reveals a sharp distribution of rates, which effectively may be represented by a single spin relaxation rate $\lambda_{\mathrm{LF}}$ for the entire spin system, while smaller values, $n<1$, suggests a distribution of rates which rapidly broadens with decreasing $n$. Only two parameters are needed to fully describe the distribution of rates: The (stretched) exponent $n$ characterizes the width of the distribution and $1 / \lambda_{\mathrm{LF}}$ is the time needed for the initial asymmetry $A$ to decay to $1 / e$ of its initial value (independent of $n$ ).

The results of the temperature dependence of $\lambda_{\mathrm{LF}}$ and $n$ are summarized in Figs. 7(a) and 7(b). At high temperatures, $T>$ $30 \mathrm{~K}$, the data are well fit with a stretched exponent $n \approx 1$, signaling that the system is rather homogeneous. Below $30 \mathrm{~K}$, with decreasing temperature, $\lambda_{\mathrm{LF}}(T)$ displays a substantial increase suggesting a rapid slowing down of the dynamics of the Co spin fluctuations. These are considered to be in the "fast fluctuation regime" at all temperatures for these particular measurements, $T>8 \mathrm{~K}$. At the same time the system becomes more inhomogeneous, as judged by the rapid decrease of $n$. At $100 \mathrm{~K}, n \approx 0.9$, but just above $T_{N}, n \approx 0.5$. The increase of $\lambda_{\mathrm{LF}}$ with decreasing $T$ is associated with the increase of AF correlations among Co moments within the individual screw chains. This claim obtains support from previous findings based on results for $\chi(T)$. A broad maximum in $\chi(T)$ signals the onset of intrachain magnetic correlations. ${ }^{8}$ An illustration of the inhomogeneity of the system at low temperatures is given by the distribution of rates depicted in the inset of Fig. 7(a). 

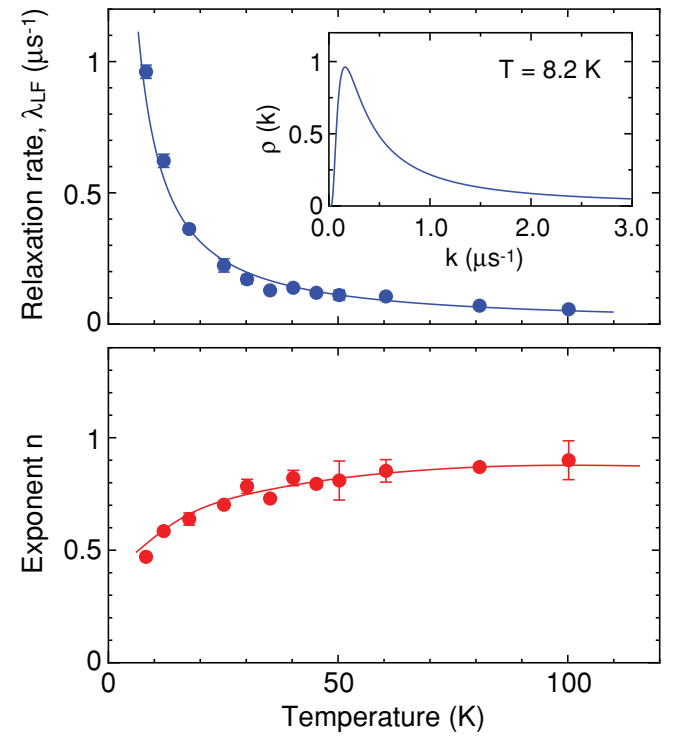

FIG. 7. (Color online) Temperature dependence of muon spin relaxation rate $\lambda_{\mathrm{LF}}$ (a, top figure) and stretched exponent $n$ (b, bottom figure) obtained in a external longitudinal field of $20 \mathrm{G}$ in the paramagnetic state. The inset in (a) depicts the distribution of relaxation rates at $T \approx 8 \mathrm{~K}$, where $n=0.5$ (see text).

Stretched exponential relaxation appears in a wide variety of phenomena, ${ }^{29}$ including spin glasses and AF systems with frustrated interactions. In such systems, the relaxation at high temperatures follows an exponential form and, as the temperature decreases (towards the freezing temperature in spin glasses), the functional form changes into a stretched exponential function ${ }^{30}$

$$
G_{z}(t)=A \exp \left[-(\lambda t)^{\frac{d}{d+2}}\right] .
$$

Here the stretched exponent depends on the effective dimensionality $d$. Qualitatively this general picture applies to our system with a reduced dimensionality, since already at $8.2 \mathrm{~K}$, Fig. 7(b) shows that $n \leqslant 0.5$, clearly below the expectations for a 3D case $(n \leqslant 3 / 5)$. There is a clear trend of decreasing $n$ with decreasing temperature. At low temperatures the tendency seems to be $n \rightarrow 1 / 3$; that is, toward the $d=1$ case. But this trend changes abruptly upon ordering, where the effective dimensionality of the system suddenly transforms into 3D.

In Fig. 8 we present the ZF- $\mu$ SR time spectrum at $T=$ $1.6 \mathrm{~K}$, deep in the AF state. Here a spontaneous Larmor precession is revealed by the time evolution of the asymmetry. The prominent structures revealed in this plot signal the presence of several inequivalent muon sites with nonvanishing internal static fields. This is characteristic of a magnetically ordered state.

The muon spectrum below $T_{N}$ is well described by assuming four components with different relaxation rates and different Larmor frequencies:

$$
G_{z}(t)=A \exp (-\lambda t)+\sum_{i=1}^{4} A_{i} \exp \left(-\lambda_{i} t\right) \cos \left(2 \pi v_{i} t+\phi\right),
$$

where the first term, a signal with vanishing Larmor frequency, is associated with muons whose spins are oriented along the

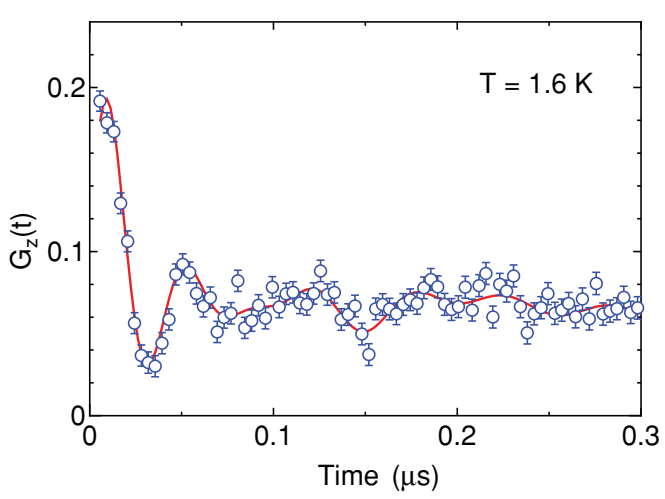

FIG. 8. (Color online) $\mu$ SR time spectrum measured in zero field in the AF state at $1.6 \mathrm{~K}$. The solid line represents a fit to the data using 5 different components.

internal static fields. This corresponds to $1 / 3$ of the total signal for our polycrystalline sample [i.e., $A \approx 1 / 3\left(A+A_{1}+A_{2}+\right.$ $\left.\left.A_{3}+A_{4}\right)\right]$. Therefore, the contribution to the asymmetry from muon sites with vanishing static magnetic dipolar field must be small, otherwise the fraction of $A$ relative to the total asymmetry would be larger. The signals with nonvanishing Larmor frequencies: $v_{1}=5.7, v_{2}=11.1, v_{3}=18.4$, and $v_{4}=$ $23.9 \mathrm{MHz}$, at $1.6 \mathrm{~K}$, arise from muons stopped at sites with internal static fields of $0.42,0.82,1.36$, and $1.76 \mathrm{kOe}$, respectively. The largest asymmetry is 0.12 , corresponding to $v_{4}$, and is substantially larger than the other signals $\left(A_{4} \gg\right.$ $A_{1} \sim A_{2} \sim A_{3}$ ). Muon motion in the crystal may be ruled out by the lack of appreciable temperature-induced changes in the asymmetries $A_{1}, A_{2}, A_{3}$, and $A_{4}$ below $T_{N}$.

The model described by Eq. (5) is plausible. For instance, the largest relaxation rate corresponds to the largest internal fields; $\lambda_{4} \approx 28 \mathrm{MHz}$. The other three have substantially smaller relaxation rates, of the order of $\lambda_{1} \sim \lambda_{2} \sim \lambda_{3} \sim 7$ $\mathrm{MHz}$. The lowest rate corresponds to the signal with vanishing Larmor frequency $\lambda<0.8 \mathrm{MHz}$. Although the model yields good fits to the data, it has limitations; namely, it is difficult to identify the muon sites. We found several interstitial sites $(x, y, z)$ with $x / a, y / b, z / c=\{0,0.5\}$, where the electric

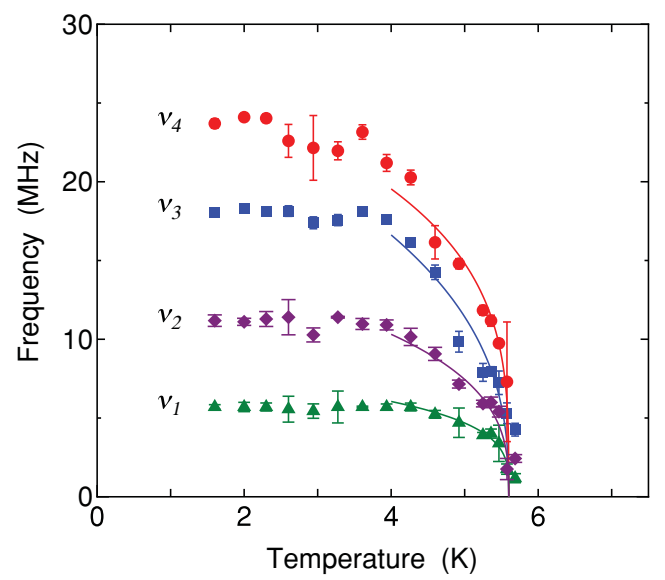

FIG. 9. (Color online) Temperature dependence of nonzero muon relaxation frequencies inferred from the zero-field data in the AF state. All the frequencies vanish at $T_{N}=5.4 \mathrm{~K}$. The solid lines represent fits to the data using the $3 \mathrm{D}$ Ising model. 
field vanishes. However, at these sites the calculated dipolar magnetic field also vanishes and, therefore, they are unlikely to contribute to the observed asymmetry. Muon sites where the dipolar magnetic field correspond to the inferred internal static fields are, for instance, near the centers of sides and faces of some $\mathrm{BaO}_{4}$ tetrahedra. However, at these places the electric field does not vanish. We conclude that most likely the observed signals must arise from muon bound to oxygen ions.

In Fig. 9 we display the temperature dependence of the nonzero muon Larmor frequencies inferred from the zero-field data. All the frequencies vanish as $T$ approaches $T_{N}$. The solid lines represent fits to the data using the critical behavior $v=$ $v_{0}\left[1-\left(T / T_{N}\right)^{\beta}\right]$. Experimentally one finds $\beta \sim 0.28$, similar to the value obtained in the analysis of the neutron diffraction data and close to the expectations of the 3D Ising model. Therefore, below $T_{N}$, the data consistently suggest that the ordered moments of $\mathrm{BaCo}_{2} \mathrm{~V}_{2} \mathrm{O}_{8}$ may be considered as a $3 \mathrm{D}$ Ising system.

In summary, as $\mathrm{BaCo}_{2} \mathrm{~V}_{2} \mathrm{O}_{8}$ approaches the antiferromagnetic state with decreasing temperatures: (i) the Co moment fluctuations undergo a drastic slowing down and (ii) the system, as probed by the muon relaxation, becomes gradually inhomogeneous. These features reflect the development of 1D intrachain AF correlations and show that, just above $T_{N}$, the dynamics of the spin system may be thought of as a disordered Ising system with reduced dimensionality (probably 1D). The phase transition at $T_{N}$ may be thought of as the order-disorder transition of $1 \mathrm{D}$ objects. Upon ordering, the dimensionality of system changes to 3 . In the AF state, near $T_{N}$, the temperature dependence of the internal static fields follows the expectations of a 3D Ising model with an effective $S=1 / 2$. The magnetic structure is characterized by a wave-vector $\vec{k}_{\mathrm{AF}}=(0,0,1)$, independent of temperature and external magnetic field. The moments of $2.18 \mu_{B}$ per Co ion are arranged AF within the screw chains, along the $c$ axis, and in the basal planes they form ferromagnetic zigzag paths along one of the axes and antiferromagnetic paths along the orthogonal (equivalent) direction.

\section{ACKNOWLEDGMENTS}

This work is based on measurements performed at the laboratory for neutron scattering at the Swiss Neutron Source SINQ and at the Swiss Muon Source, PSI, Villigen, Switzerland. This work was partially supported by the Danish Natural Science Research Council under DANSCATT (N. C.) and by the Advanced International Education and Research Support Program from the Ministry of Education, Culture, Sports, Science and Technology (MEXT) of Japan (Y. K.).
${ }^{1}$ Order and Disorder in Quantum Spin Systems, edited by T. Tonegawa, S. Miyashita, and H. Takayama, Suppl. 145 (Progress of Theoretical Physics, Japan, 2002).

${ }^{2}$ F. D. M. Haldane, Phys. Rev. Lett. 45, 1358 (1980).

${ }^{3}$ F. D. M. Haldane, Phys. Rev. B 25, 4925 (1982).

${ }^{4}$ H. A. Bethe, Z. Phys. 71, 205 (1931).

${ }^{5}$ G. Gomez-Santos, Phys. Rev. B 41, 6788 (1990).

${ }^{6}$ Y. Uchiyama, Y. Sasago, I. Tsukada, K. Uchinokura, A. Zheludev, T. Hayashi, N. Miura, and P. Böni, Phys. Rev. Lett. 83, 632 (1999).

${ }^{7}$ Z. He, D. Fu, T. Kyômen, T. Taniyama, and M. Itoh, Chem. Mater. 17, 2924 (2005)

${ }^{8}$ Z. He, T. Taniyama, and M. Itoh, J. Cryst. Growth 289, 734 (2006). ${ }^{9}$ R. Wichmann and Hk. Müller-Buschbaum, Z. Anorg. Allg. Chem. 534, 153 (1986).

${ }^{10}$ S. Kimura, H. Yashiro, M. Hagiwara, K. Okunishi, K. Kindo, Z. He, T. Taniyama, and M. Itoh, J. Phys.: Conference Series 51, 9910 (2006).

${ }^{11}$ S. Kimura, H. Yashiro, K. Okunishi, M. Hagiwara, Z. He, K. Kindo, T. Taniyama, and M. Itoh, Phys. Rev. Lett. 99, 087602 (2007).

${ }^{12}$ Z. He, T. Taniyama, and M. Itoh, Appl. Phys. Lett. 88, 132504 (2006).

${ }^{13}$ Z. He, T. Taniyama, T. Kyômen, and M. Itoh, Phys. Rev. B 72, 172403 (2005).

${ }^{14}$ H.-J. Mikeska, Asimkumar Ghosh, and A. K. Kolezhuk, Phys. Rev. Lett. 93, 217204 (2004).
${ }^{15}$ K. Okunishi and T. Suzuki, Phys. Rev. B 76, 224411 (2007).

${ }^{16}$ S. Kimura, T. Takeuchi, K. Okunishi, M. Hagiwara, Z. He, K. Kindo, T. Taniyama, and M. Itoh, Phys. Rev. Lett. 100, 057202 (2008).

${ }^{17}$ S. Kimura, M. Matsuda, T. Masuda, S. Hondo, K. Kaneko, N. Metoki, M. Hagiwara, T. Takeuchi, K. Okunishi, Z. He, K. Kindo, T. Taniyama, and M. Itoh, Phys. Rev. Lett. 101, 207201 (2008).

${ }^{18}$ S. Suga, J. Phys. Soc. Jpn. 77, 074717 (2008).

${ }^{19}$ Y. Ideta, T. Ohno, Y. Kishimoto, Y. Kawasaki, Y. Michihiro, Z. He, Y. Ueda, and M. Itoh (unpublished).

${ }^{20}$ C. N. Kuo, C. S. Lue, Z. Z. He, and M. Itoh, Solid State Commun. 149, 341 (2009).

${ }^{21}$ N. M. Bogoliubov, A. G. Izergin, and V. E. Korepin, Nucl. Phys. B 275, 687 (1986).

${ }^{22}$ J. Rodriguez-Carvajal, Physica B 192, 55 (1993).

${ }^{23}$ Magnetic Properties of Layered Transition Metal Compounds, edited by L. J. de Jongh (Klewer, Dordrecht, London, 1990).

${ }^{24}$ M. F. Collins, Magnetic Critical Scattering (Oxford University Press, New York 1989).

${ }^{25}$ Y. Ozeki and N. Ito, J. Phys. A: Math. Theor. 40, R149 (2007).

${ }^{26}$ M. E. Fisher, Rep. Prog. Phys. 30, 615 (1967).

${ }^{27}$ R. S. Hayano, Y. J. Uemura, J. Imazato, N. Nishida, T. Yamazaki, and R. Kubo, Phys. Rev. B 20, 850 (1979).

${ }^{28}$ A. Keren, Phys. Rev. B 50, 10039 (1994).

${ }^{29}$ J. C. Phillips, Rep. Prog. Phys. 59, 1133 (1996).

${ }^{30}$ J. C. Phillips, J. Non-Cryst. Solids 182, 155 (1995). 